\title{
Study on the Age of SARS-CoV-2 in Respiratory and Digestive Tract Samples: A Retrospective Study guixian-wu1,dongqin-Iv*,susu-He,qian-chen,lin- ling,shaungquan-yan,xiaomai-wu,yongpo-jiang
}

\section{Guixian Wu}

Taizhou Enze Medical Center\&Taizhou Hospital affiliated toZhejian University

\section{Susu He}

Taizhou Enze Medical Center \&Taizhou Hospital affiliated to Zhejiang University

\section{Qian Chen}

Taizhou Enze Medical Center\&Taizhou Hospital affiliated to Zhejiang University

\section{Lin Ling}

Taizhou Enze Medical Center \&Taizhou Hospital affiliated to Zhejiang University

\section{Shuangquan Yan}

Taizhou Enze Medical Center Enze Hospital

\section{Xiaomai Wu}

Taizhou Enze Medical Center\&Taizhou Hospital affiliated to Zhejiang University

\section{Yongpo Jiang}

Taizhou Enze Medical Center \&Taizhou Hospital affiliated to Zhejiang University

Dongqing Lv ( $\nabla$ lvdq@enzemed.com )

Taizhou Hospital of Zhejiang Province

Research article

Keywords: SARS-CoV-2; SARS-CoV-2 existence time; sputum; feces; nasopharyngeal swab; upper respiratory tract; lower respiratory tract; digestive tract.

Posted Date: April 30th, 2020

DOl: https://doi.org/10.21203/rs.3.rs-21793/v1

License: (1) This work is licensed under a Creative Commons Attribution 4.0 International License. Read Full License 


\section{Abstract}

Background: An acute respiratory disease, caused by a novel coronavirus (SARS-CoV-2, previously known as 2019-nCoV), the coronavirus disease 2019 (COVID-19), has spread throughout China and received worldwide attention. After nearly 2 months efforts by the Chinese government and people from all walks of life, the epidemic in China has gradually been controlled. The awareness of the disease has gradually increased. We have detected the SARS-CoV-2 gene in the patient's sputum, throat swabs and feces, blood and even urine samples, but whether the SARS-CoV-2 RNA is detected in the upper and lower respiratory tracts and the extrapulmonary age is unknown.

Methods: We collected data from 146 patients admitted to the Public Health Center of Taizhou Hospital, Zhejiang University, China from January 23, 2020 to March 11, 2020, and confirmed the diagnosis of new-type coronavirus pneumonia by RT-PCR. The final selection was made after screening. For a total of 134 confirmed patients, we collected their clinical data and sputum, nasopharyngeal swabs or throat swabs, fecal nucleic acid test results, and calculated the age of each specimen.

Results: A total of 134 confirmed patients were included in the study; of them, 93 were of the common type, and 41 were critically ill. There were no significant differences between males and females, $P=$ $0.853)$ and their epidemiological history $(P=0.441)$. The average age of severe patients was $55.85 \pm$ 13.28 years, which was higher than that of common patients at $44.06 \pm 12.86$ years $(P=0.00)$. The results showed that the time of SARS-CoV-2 RNA in the upper respiratory tract was $15.1 \pm 7.23$ days for common patients and $20.56 \pm 6.59$ days for severe patients, which was longer than that of common patients $(P=0.00)$. The time of SARS-CoV-2 RNA in the lower respiratory tract was $27.45 \pm 10.06$ days for common patients and $29.78 \pm 10.11$ days for severe patients. There was no statistical difference $(P=$ 0.328 ); SARS-CoV-2 RNA existed in the digestive tract. The time was $22.6 \pm 7.69$ days for common patients and $27.24 \pm 7.86$ days for severe patients, which was longer than that of common patients $(P=$ $0.01)$. SARS-CoV-2 existed for a shorter time in the upper respiratory and digestive tracts than in the lower respiratory tract.

CONCLUSION: SARS-CoV-2 is present in the lower respiratory tract for longer than the upper respiratory and digestive tracts, and there were no statistical differences in the lower respiratory tract between common and severe patients.

\section{Background}

In December 2019, a new type of coronavirus was discovered in Wuhan for the first time and quickly spread to the whole country and the world[1]. As of March 28, 2020, a total of 82,214 cases were diagnosed in China, with 3,791 deaths, and 75,122 patients have been cured. A series of preventive control and medical rescue measures have prevented an increase in the epidemic situation in China, and the epidemic situation has eased in most provinces. As of March 11, 2020, all 146 confirmed patients in the author's area were cured and discharged. By March 28, 2020, 1,251 cases were confirmed in Zhejiang 
Province, with 0 deaths, and 1,225 patients were cured; the remaining 16 were mostly imported cases. To date, the gold clinical diagnostic method for SARS-CoV-2 is to detect the viral nucleic acid by RT-PCR in a sample from the nasal cavity, throat or other respiratory tract and to further confirm these results by nextgeneration sequencing[2].However, Chinese researchers have detected SARS-CoV-2 virus nucleic acid in the faeces of several confirmed cases of SARS-CoV-2[3] and in the urine and blood[4] of severely ill patients, suggesting the possible existence of the SARS-CoV-2 faecal-oral and blood transmission routes[5]. To reduce the chance of human-to-human transmission, we performed multiple nucleic acid tests on sputum, faeces, throat swabs or nasopharyngeal swabs for each patient until we ensured that the viruses in the patients' upper and lower respiratory and digestive tracts had been removed. Although people have gradually deepened their understanding of the clinical manifestations, pathology, and experience of diagnosis and treatment, the duration of SARS-CoV-2 RNA detection in upper and lower respiratory tracts and extrapulmonary specimens is unclear, and the existence time of SARS-CoV-2 in each specimen is important for the epidemic situation. Prevention and control are of great significance.

Our goal is to determine the existence time of SARS-CoV-2 in the upper, lower, and digestive tracts and compare their differences.

\section{Methods}

We retrospectively analysed the detoxification time of 146 patients with SARS-CoV-2 diagnosed by RTPCR in the public health centre of Taizhou Hospital affiliated with Zhejiang University in Zhejiang Province from December 2019 to March 2020. A total of 134 confirmed patients were diagnosed, and the patients were divided into common and severe types according to the $\square$ Diagnosis and Treatment Protocol for Novel Coronavirus Pneumonia (Trial Version 7) $₫$ [1]. During hospitalization, these patients were discussed by a group composed of experts in respiratory medicine, ICU, radiology, etc. The main outcome of this study was to calculate the time difference from the onset to the first negative as the time of existence. The first negative time is defined as the first negative time after $2-3$ consecutive negative nucleic acid tests. For a small number of asymptomatic patients, the difference between the date of diagnosis and the negative time of the first negative nucleic acid test is used as the time of existence. The time difference between the date and the first negative nucleic acid test was used as the time of existence. The common type is defined as fever, respiratory tract symptoms, and pneumonia on imaging; severe patients are defined as meeting any of the following criteria: 1 . Shortness of breath, breathing $\geq 30$ beats/min; 2 . In the resting state, oxygen saturation $\leq 93 \%$; 3 . Arterial blood oxygen partial pressure (PaO2)/oxygen concentration ( $\mathrm{FiO} 2) \leq 300 \mathrm{mmHg}$. The time of existence was calculated as the difference between the time of onset and the time of the first negative results (the time between the first negative result after $2-3$ consecutive negative results). Time difference is the first time with negative results after $2-3$ consecutive negatives.[1]

\section{Statistical analysis}


SPSS 23 software was used for the statistical analysis. Continuity data were expressed as the mean \pm standard deviation and the median, and the categorical data were expressed as percentage of frequency; chi-squared test was used for the comparison of rates. $P<0.05$ was considered statistically significant.

\section{Results}

\section{Study population}

A total of 134 patients were selected from 146 patients with a clear diagnosis, and the reasons for exclusion included pregnancy, age under 18 years old, having incomplete samples and other reasons(Figure 1).

Demographic data, epidemiological history, symptoms and underlying diseases of patients included in the study(Table 1)

A total of 134 patients with a new type of coronavirus pneumonia diagnosed by RT-PCR were included in this study, including 93 patients with the common type (69.4\% of the total) and 41 patients with severe disease (30.6\% of the total). In terms of statistical data, disease characteristics and medication, there was no significant differences in sex and epidemiology between the common group and the severe group. The average age of the heavy group was higher than that of the general group. The age of the severe group was $55.85 \pm 14.04$ years, the age of the mild patients was $44.06 \pm 12.86$ years old, and the ratio of male to female was close to $1: 1$, suggesting that male and female susceptibility is similar. The main clinical manifestation of SARS-CoV-2 is fever (75.3\%), especially in severe cases. Patients with a fever accounted for $92.6 \%$, with body temperatures fluctuating between $38^{\circ} \mathrm{C}$ and $39{ }^{\circ} \mathrm{C}$ and 39 and $40{ }^{\circ} \mathrm{C}$ each account for $44.7 \%$ and $31.6 \%$ of patients, respectively, which is a small number of patients. The body temperature can exceed $40{ }^{\circ} \mathrm{C}(5.3 \%)$, while the common type was dominated by a low fever, most of which were between $38-39{ }^{\circ} \mathrm{C}(46 \%)$. In addition, $82 \%$ of patients with SARS-CoV-2 pneumonia may have cough, mainly with dry cough. Mainly, $38 \%$ of patients can have a small amount of sputum, mostly white sputum, and chest tightness, fatigue, and diarrhoea were also common clinical manifestations of COVID-19. It is worth noting that most patients have diarrhoea that appears during the course of the disease; in addition to considering the virus is in the digestive system, the damage factors and side effects of antiviral drugs (lopinavir/ritonavir tablets and arbidol) need to be considered. Actually, diarrhoea caused by the virus itself may be a little $23.8 \%$. In terms of the treatment, for critically ill patients, we applied small doses of hormones and gamma globulin early to prevent cytokine storms. Most of the hormones were gradually reduced after 3 days of intravenous infusion of $40 \mathrm{mg} q \mathrm{qd}$. For severe patients, the dose can be increased and the time can be extended according to the situation. The dose should not exceed $80 \mathrm{mg}$. Intravenous gamma globulin of 20-30 mg qd can be used for 3 days. The number of critically ill patients increased. With the inhalation of oxygen or high-frequency oxygen, the condition of most patients can be improved. Currently, 134 patients were included. For the 41 severely ill patients, none of them were treated with a non-invasive ventilator or tracheal intubation, and all were discharged after being cured. 
The individual existence of the virus in each sample varies greatly, ranging from 1 to 48 days (Fig. 2, Table 2). In general, SARS-CoV-2 has the longest duration in the lower respiratory tract, followed by the digestive tract. The respiratory tract has the shortest time (Fig. 3), which may be related to the pathogenic characteristics of SARS-CoV-2. SARS-CoV-2 viral particles are present for longer in the upper respiratory tract and digestive tract in severe patients than in common patients, with an average difference of approximately 4-5 days. However, there were no significant differences in the age of SARS-CoV-2 in the lower respiratory tract between light patients and severe patients (Table 2). The new type of coronavirus has a longer life in the upper respiratory tract than the common type; the common type is $15.1 \pm 7.23$ days, the longest existence time is 45 days, the heavy existence time is $20.56 \pm 6.59$ days, and the longest is 45 days. The difference between the two is statistically significant $(P=0.00)$; the time of existence of SARS-CoV- 2 in the lower respiratory tract was $27.45 \pm 10.06$ days for the common type, 46 days for the longest, $29.78 \pm 10.11$ days for the heavy, and 48 days for the longest. There was no significant difference between the two groups $(P=0.328)$; the age of the new type of coronavirus in the digestive tract was $22.6 \pm 7.69$ days for the common type and $27.24 \pm 7.86$ days for the heavy type. There was a statistically significant difference between the two groups $(P=0.01)$. The existence of a heavy digestive tract was longer in the severe type than in the common type.

\section{Discussion}

According to statistical data, SARS-CoV-2 is generally susceptible, and the proportion of males and females infected is similar, and it has little to do with whether the diseased area or contact with confirmed patients. In addition, the elderly, patients with basic diseases, and immune dysfunction more easily evolve into critically ill patients. Notably, $48.8 \%$ of patients without underlying diseases eventually evolve into severe disease, so early detection and early treatment are particularly important. SARS-CoV-2 primarily infects the lower airway and binds to ACE2 on alveolar epithelial cells. The virus activates immune cells and induces the secretion of inflammatory cytokines and chemokines into pulmonary vascular endothelial cells [6], mainly causing pneumonia. SARS-CoV-2 RNA was more readily detected in induced sputumthan in throat swabs of convalescent COVID-19 patients[7, 8]. The main clinical manifestations are respiratory symptoms, including fever $(75.3 \%)$, cough $(82 \%)$, sputum $(38 \%)$, and only a small number of gastrointestinal symptoms, including diarrhoea and bloating[2]. From our statistics, we know that the presence of the new type of coronavirus in upper respiratory tract specimens is significantly shorter. Even if 2 or more negative throat swabs or nasopharyngeal swabs are negative, lower respiratory tract specimens and faecal specimens may continue to detoxify, and these patients are often still infectious. [2] As mentioned above, the existence of SARS-CoV-2 in the lower respiratory tract is closer to the calculation of the detoxification time of SARS-CoV-2. Due to statistical data, the average lower respiratory tract virus clearance time of SARS-CoV-2 is close to one month, but there are large differences among individuals. The minimum clearance time is 1 day, and the maximum clearance time is 48 days. For the liquid test, it is recommended to use the sputum test as the standard. If the condition is limited, an upper respiratory tract specimen must be taken. After reaching the discharged specimen, it is recommended to continue to isolate the patient for 1 to 1.5 months. There was no significant difference in clearing time in 
the lower respiratory tract between common and severe patients, indicating that the severity of the disease does not affect the clearance of SARS-CoV-2 in the lung. That is, even in common patients, we still need to be vigilant with a long detoxification time; even if the patient's symptoms are relieved, and the imaging absorption is considered to be a complete cure of the patient. The detoxification time of SARSCoV-2 may continue until the imaging is completely absorbed. Among the patients we studied, at least 4 had complete absorption, but SARS-CoV-2 could still be detected in sputum. The data of one patient can be seen in Fig. 4; while in severe patients, even if the imaging has been largely absorbed, fibrotic lesions have formed, and sputum, pharyngeal swabs and SARS-CoV-2 nucleic acid can still be detected in faeces. Therefore, it is unreliable to judge the course of disease based only on the outcome of imaging results and improvement of symptoms. We still need to take the results of nucleic acid tests, especially lower respiratory tract specimens, including sputum fluid or alveolar lavage fluid; sputum specimens have the advantages of convenient material collection and reduced exposure and should be widely promoted [7]. In addition, $92.6 \%$ of severe patients used hormones, and many studies mentioned that hormone use affects virus clearance, but it can be seen that the use of hormones in small doses as appropriate does not affect virus clearance [8]. However, due to the small sample size, more data need to be clarified. Due to statistical data, the average lower respiratory tract virus clearance time of SARS-CoV-2 is close to one month, but there are large differences among individuals. The minimum clearance time is 1 day, and the maximum clearance time is 48 days.

\section{Conclusion}

SARS-COV-2 RNA more readily detected in induced sputum than in throat swabs of convalescent COVID19 patients, and there were no statistical differences in the lower respiratory tract between ordinary and severe patients.

\section{Abbreviations}

RT-PCR囚reverse transcription-polymerase chain reaction

SARS-COV-2『Severe acute respiratory syndrome coronavirus-2

\section{Declarations}

\section{Ethics approval and consent to participate}

All the datas involved in this article have obtained the oral consent of the patients.

Due to the particularity of the epidemic, no written informed consent forms was left.Ethics approval was granted by Committee of Enze Hospital of Taizhou Enze Medical Center(NO.K20200204区

\section{Consent for publication}


The pictures and personal information in the article have been approved by the patients

\section{Availability of data and material}

In order to protect patients' privacy,we will not deposit our datasets in publicly available repositories. people who need the raw datas can contact the Corresponding author.

\section{Funding}

This work was funded by The Science and Technology Project of Taizhou(1902kynology) .

\section{Competing interests}

The authors declare no competing interests.

\section{Authors' contributions}

DL contributed to the study design and data interpretation. GW contributed to data analysis, data interpretation and writing of the manuscript. GW,XW,QC,SHe,YJ, LL and SY contributed to clinical management, patients' data collection. SY,LL,YJ contributed to critical revision of the manuscript. All authors reviewed and approved the final version of the manuscript.

\section{Acknowledgements}

We thank the CDCs of Taizhou City and zhejian Province for helping with confirmation of the diagnosis of the viral infection.

\section{Author information}

\section{Affiliations}

Division of Respiratory Medicine, Taizhou Enze Medical Center \& Taizhou hospital affiliated to Zhejiang university, Taizhou, Zhejiang, 318000, China

Guixian Wu,Lin-Ling,Xiaomai Wu

Division of Respiratory Medicine, Taizhou Enze Medical Center Enze hospital, Taizhou, Zhejiang, 318000, China

Shuangquan Yan,Dongqing Lv

Division of Intensive care medicine, Taizhou Enze Medical Center \& Taizhou hospital affiliated to Zhejiang university, Taizhou, Zhejiang, 318000, China

Yongpo Jiang 


\section{Corresponding authors}

Correspondence to Dongqing Lv Email: Ivdq@enzemed.com

\section{References}

1. Medicine, N.H.C.N.A.o.T.C., Diagnosis and Treatment Protocol for NovelCoronavirus Pneumonia (Trial Version 7). Chinese medical journal, March 3, 2020: p. Chin Med J, 2020,133:Epub ahead of print.

2. Jiang, F., et al., Review of the Clinical Characteristics of Coronavirus Disease 2019 (COVID-19). J Gen Intern Med, 2020

3. Huaxia, Chinese researchers isolate novel coronavirus strain from feces. Xinhuanet, Feb 13, 2020

4. Chen, W., et al., Detectable 2019-nCoV viral RNA in blood is a strong indicator for the further clinical severity. Emerg Microbes Infect, 2020. 9(1): p. 469-473.

5. W. Guan, Z.N., Yu Hu, W. Liang, C. Ou, J. He, L. Liu, H. Shan, C. Lei, D.S.C. Hui,, et al., Clinical Characteristics of Coronavirus Disease 2019 in China. The New England Journal of Medicine 202002-28: p. 497-506.

6. Singhal, T., A Review of Coronavirus Disease-2019 (COVID-19). Indian J Pediatr, 2020.

7. Zou L, R.F., Huang M, Liang L, Huang H, Hong Z, Yu J, Kang M, Song Y, Xia J, Guo Q, Song T, He J, Yen HL, Peiris M, Wu J, SARS-CoV-2 viral load in upper respiratory specimens of infected patients. N Engl J Med, : 25 February 2020.

8. *Huanqin Han, Q.L., Fan Mo, and W.Z. Lieming Long, SARS-CoV-2 RNA more readily detected in induced sputum than in throat swabs of convalescent COVID-19 patients. Lancet Infect Dis 2020, March 12, 2020(https://doi.org/10.1016/ S1473-3099(20)30174-2).

\section{Tables}

Due to technical limitations the Tables are available as a download in the Supplementary Files.

\section{Figures}




\begin{tabular}{|c|c|}
\hline & $\begin{array}{l}146 \text { patients with } \\
\text { SARS-COV-2 confirmed } \\
\text { hv nucleir acid tecting }\end{array}$ \\
\hline $\begin{array}{l}1 \text { pregnant woman } \\
3 \text { under } 18 \mathrm{Y} \\
\text { Incomplete test data for } \\
\text { patients and other reasons }\end{array}$ & 8 \\
\hline & $\begin{array}{l}134 \text { patients with confirmed } \\
\text { SARS-COV- } 2 \text { were included } \\
\text { in the study }\end{array}$ \\
\hline
\end{tabular}

\section{Figure 1}

Flow chart of the study population

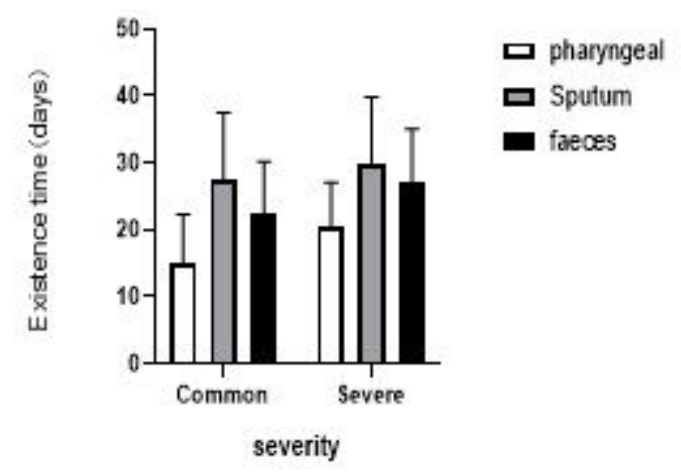

Figure 2

Comparison of the existence time of SARS-COV-2 in each specimen $₫ S D \pm S t a n d a r d$ deviation $\rrbracket$

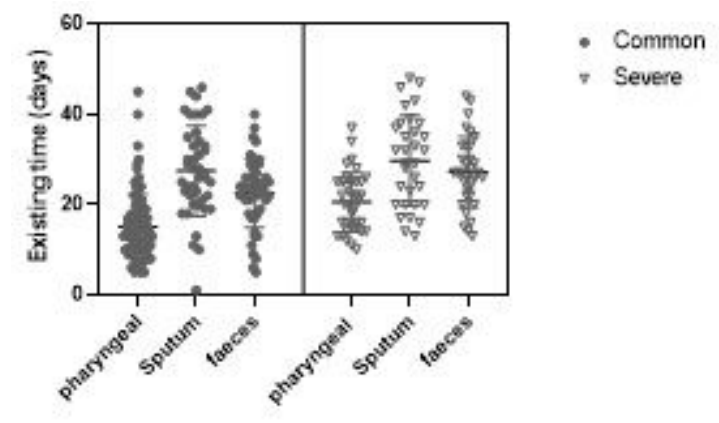

Figure 3

Comparison of the existence time of SARS-COV-2 in each specimen $\varangle \mathrm{SD} \pm$ Standard deviation $\rrbracket$ 


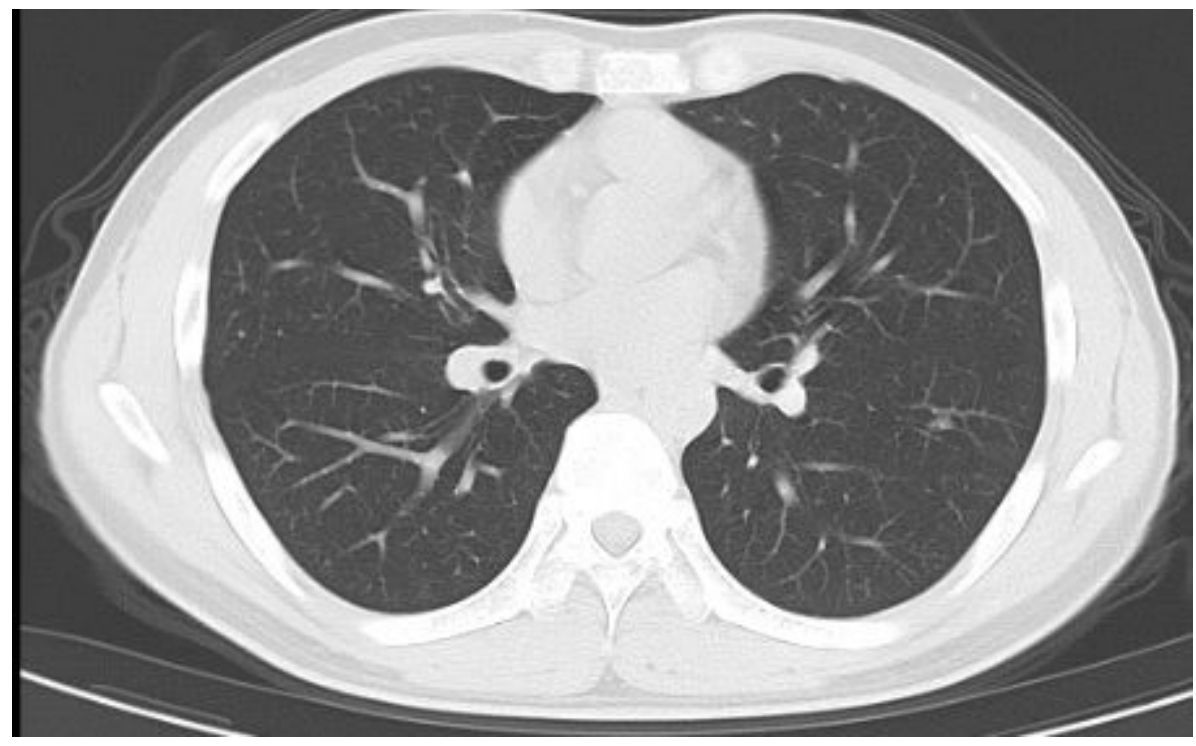

\section{Figure 4}

Patient 1: A young common type patient, who was still detected sars-cov-2 virus by rRT-PCR with intrapulmonary absorption and remission of symptoms. Figure 4 is the chest CT result on the first day of onset, suggesting a left lung patch shadow, nodule shadow

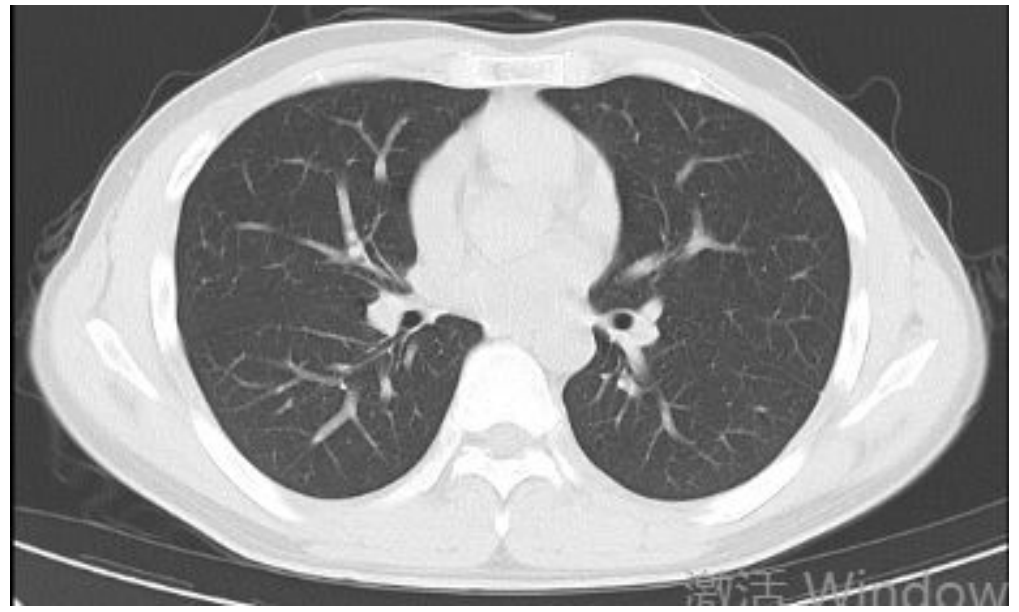

Figure 5

Patient 1: A young common type patient, who was still detected sars-cov-2 virus by rRT-PCR with intrapulmonary absorption and remission of symptoms. Figure 4 is the chest CT result on the first day of onset, suggesting a left lung patch shadow, nodule shadow. Figure 5 imaging results on the 13th day of onset shows that the lesion was completely absorbed; the presence of coronavirus in the upper respiratory tract (pharyngeal swab) lasted for 7 days, the lower respiratory tract for 36 days, and the digestive tract 30 days. The symptoms of the patient lasted 10 days. 


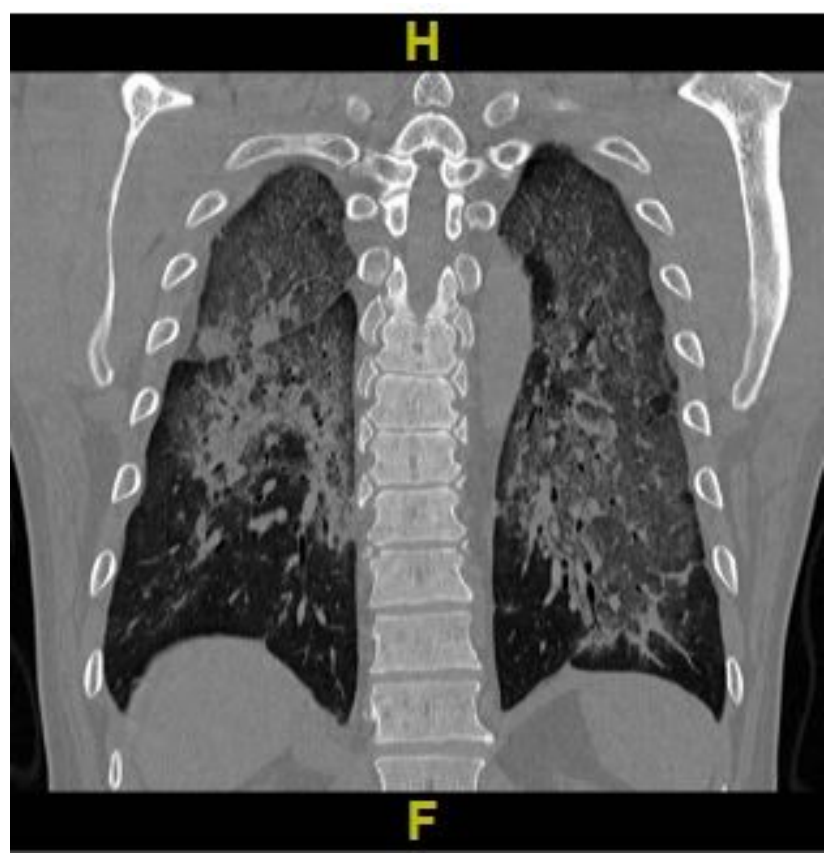

\section{Figure 6}

Patient 2: An old diagnosed patient progressed from normal to severe. Figures 6, 7, 8, and 9 are the imaging manifestations at 1 week of onset: large ground glass opacity and patches on both lungs, shadow, paving stone disease, and fiber cord-like shadows, some of which can be consolidated. 


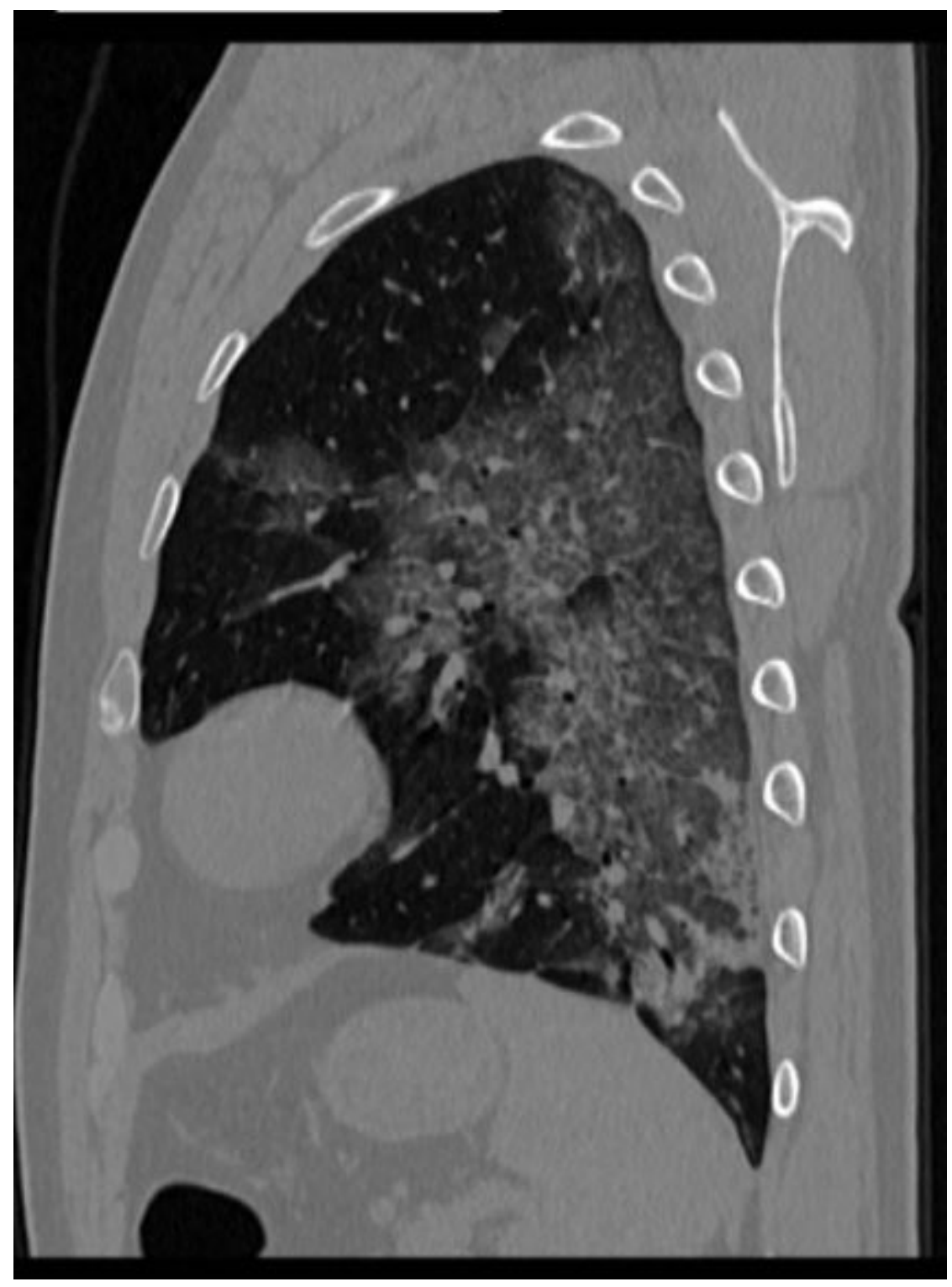

\section{Figure 7}

Patient 2: An old diagnosed patient progressed from normal to severe. Figures $6,7,8$, and 9 are the imaging manifestations at 1 week of onset: large ground glass opacity and patches on both lungs, shadow, paving stone disease, and fiber cord-like shadows, some of which can be consolidated. 


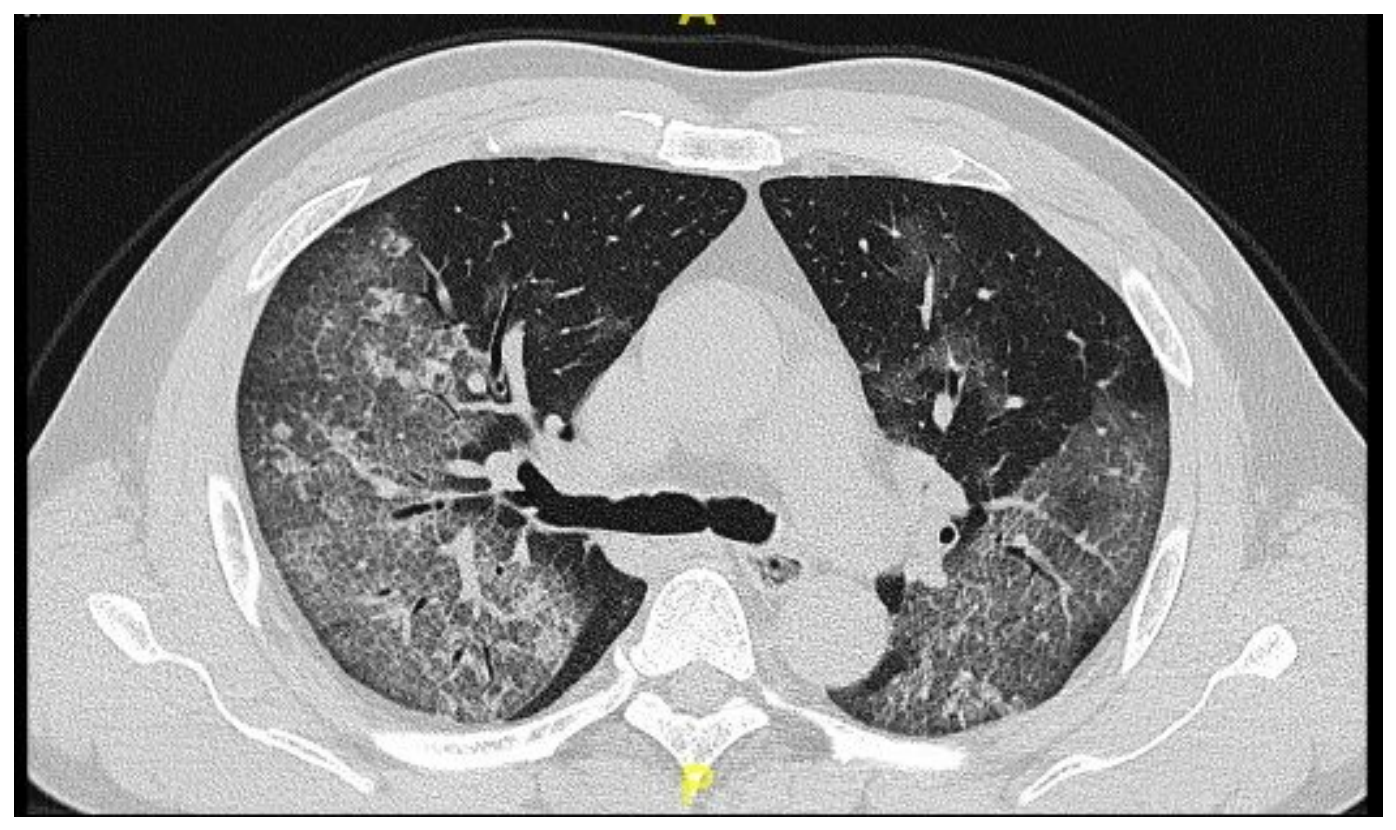

Figure 8

Patient 2: An old diagnosed patient progressed from normal to severe. Figures $6,7,8$, and 9 are the imaging manifestations at 1 week of onset: large ground glass opacity and patches on both lungs, shadow, paving stone disease, and fiber cord-like shadows, some of which can be consolidated.

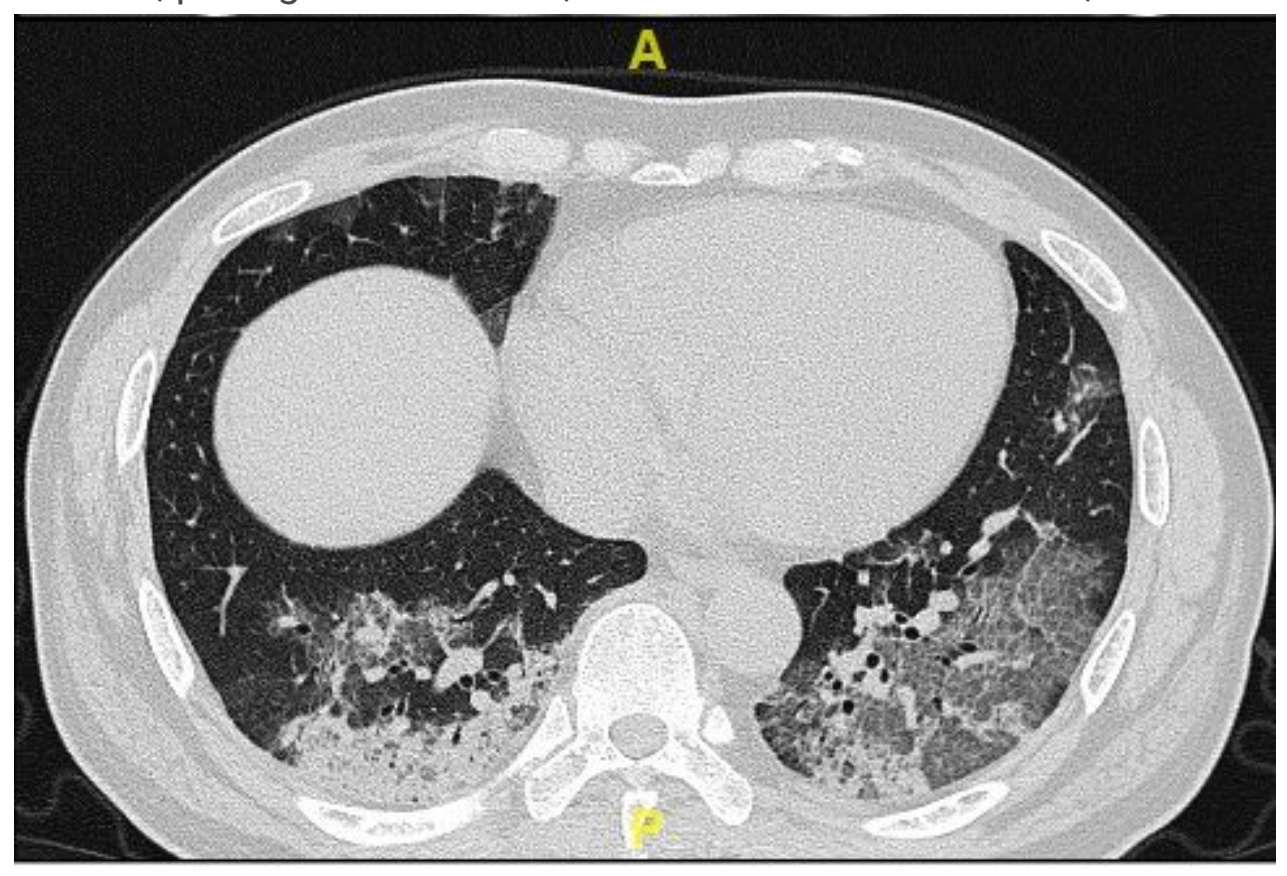

\section{Figure 9}

Patient 2: An old diagnosed patient progressed from normal to severe. Figures 6, 7, 8, and 9 are the imaging manifestations at 1 week of onset: large ground glass opacity and patches on both lungs, shadow, paving stone disease, and fiber cord-like shadows, some of which can be consolidated. 


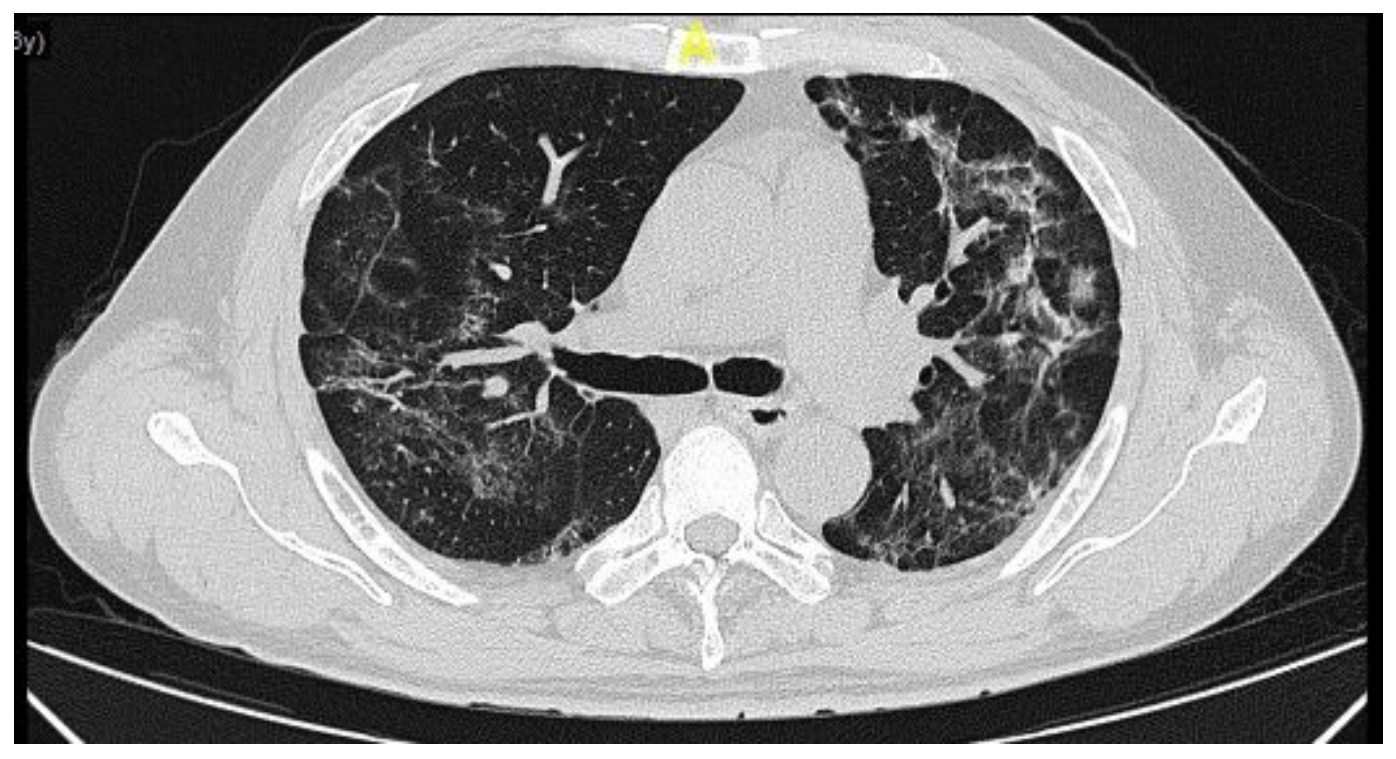

Figure 10

Patient 2: An old diagnosed patient progressed from normal to severe. Figures 10 and 11 are the results of chest CT in January. It is significantly absorbed than before, leaving fiber cord shadows. SARS-CoV-2 RNA was present in the patient in the upper respiratory tract for 37 days, the lower respiratory tract (sputum) for 36 days, and the digestive tract (faeces) for 37 days.

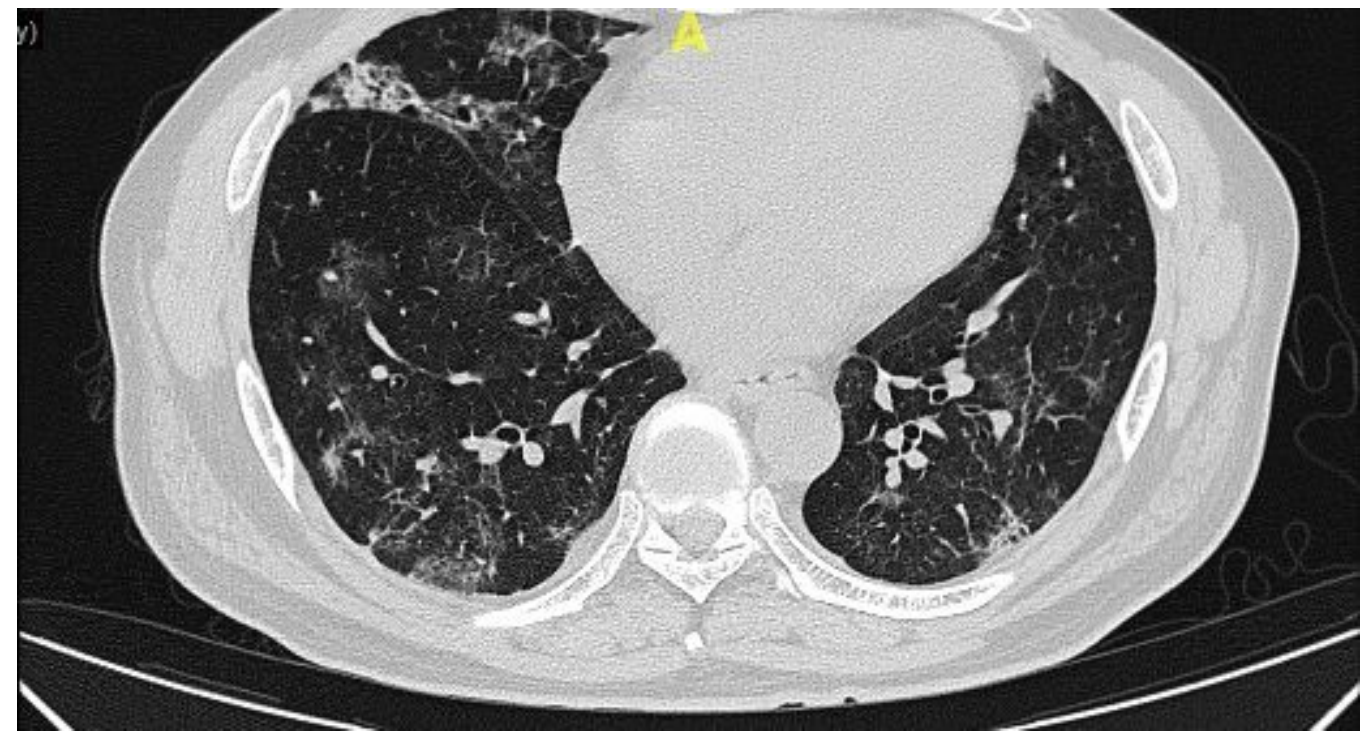

Figure 11

Patient 2: An old diagnosed patient progressed from normal to severe. Figures 10 and 11 are the results of chest CT in January. It is significantly absorbed than before, leaving fiber cord shadows. SARS-CoV-2 RNA was present in the patient in the upper respiratory tract for 37 days, the lower respiratory tract (sputum) for 36 days, and the digestive tract (faeces) for 37 days.

\section{Supplementary Files}


This is a list of supplementary files associated with this preprint. Click to download.

- Tables.pdf 